\title{
Virulencia y variabilidad de Porphyromonas gingivalis y Aggregatibacter actinomycetemcomitans y su asociación a la periodontitis
}

\author{
Virulence and variability on Porphyromonas gingivalis and Aggregatibacter \\ actinomycetemcomitans and their association to periodontitis \\ Díaz Zúñiga J11, Yáñez Figueroa J¹, Melgar Rodríguez S1, Álvarez Rivas C1, \\ Rojas Lagos $\mathrm{C}^{1}$, Vernal Astudillo $\mathrm{R}^{1}$
}

\begin{abstract}
RESUMEN
Las periodontitis son un conjunto de patologías de naturaleza inflamatoria y etiología infecciosa producidas por el biofilm patogénico subgingival. Porphyromonas gingivalis y Aggregatibacter actinomycetemcomitans son bacterias periodonto-patógenas que pueden causar daño directo a las estructuras periodontales a través de los diversos factores de virulencia que expresan. Sobre la base de estos factores de virulencia, distintos genotipos y serotipos bacterianos se han descrito, cada uno de ellos con una potencial variable patogenicidad. En esta revisión bibliográfica se describen diferentes factores de virulencia de $P$. gingivalis y $A$. actinomycetemcomitans y se discute la variable inmunogenicidad y patogenicidad de los distintos genotipos y serotipos descritos para ellos. Tanto $P$. gingivalis como $A$. actinomycetemcomitans poseen diversos factores de virulencia asociados al inicio, progresión y severidad de las periodontitis. En P. gingivalis, los factores de virulencia para los cuales se describen distintos genotipos y/o serotipos son fimbria, LPS y cápsula bacteriana, y en A. actinomycetemcomitans son leucotoxina A, Cdt y LPS. Cada uno de estos distintos genotipos y serotipos induce una respuesta inmuno-inflamatoria diferente en el hospedero y, por lo tanto, se podrían asociar a una variable patogenicidad y podrían determinar las características clínicas de la enfermedad.
\end{abstract}

Rev. Clin. Periodoncia Implantol. Rehabil. Oral Vol. 5(1); 40-45, 2012.

Palabras clave: Virulencia, genotipos, serotipos, Porphyromonas gingivalis, Aggregatibacter actinomycetemcomitans.

\section{ABSTRACT}

Periodontitis represents a heterogenic group of periodontal infections elicited by bacteria residing at the subgingival biofilm. Although this biofilm is constituted by a broad variety of bacterial species, only a limited number has been associated with the periodontitis aetiology, among them Porphyromonas gingivalis and Aggregatibacter actinomycetemcomitans. Both $P$. gingivalis and $A$. actinomycetemcomitans express a number of virulence factors that contribute to direct tissue damage and, based on them, distinct genotypes and serotypes have been described, each one with a potential variable pathogenicity. This review aimed to analyze the different virulence factors described for $P$. gingivalis and $A$. actinomycetemcomitans and to discuss the variable immunogenicity and pathogenicity of their serotypes and genotypes. $P$. gingivalis and $A$. actinomycetemcomitans express different virulence factors and they determine the initiation, progression, and severity of periodontitis. In $P$. gingivalis, distinct serotypes and/or genotypes are described based on fimbriae, LPS, and capsule. Additionally, in A. actinomycetemcomitans distinct serotypes and/or genotypes are described based on leucotoxin A, Cdt, and LPS. These distinct serotypes and genotypes induce a differential immunoinflammatory response and, thus, could be associated with variations in pathogenicity and reflected in clinic characteristics of the disease.

Rev. Clin. Periodoncia Implantol. Rehabil. Oral Vol. 5(1); 40-5, 2012.

Key words: Virulence, genotypes, serotypes, Porphyromonas gingivalis, Aggregatibacter actinomycetemcomitans.

\section{INTRODUCCIÓN}

Las periodontitis son un conjunto de patologías de naturaleza inflamatoria y etiología infecciosa producidas por el biofilm subgingival que coloniza el surco gingivodentario(1). Se caracterizan por la destrucción del soporte periodontal, que está conformado por el ligamento periodontal, el cemento radicular y el hueso alveolar, y eventualmente provocan la pérdida de los dientes ${ }^{(1)}$. En términos generales, son una de las patologías de mayor prevalencia en seres humanos y, además del daño local que inducen, son un factor modificante de la salud general del individuo(2).

Las bacterias periodontopatógenas son los agentes etiológicos de las periodontitis ${ }^{(3)}$, entre ellas, Porphyromonas gingivalis y Aggregatibacter actinomycetemcomitans han sido ampliamente asociadas al inicio, progresión y severidad de las enfermedades periodontales $^{(3,4,5)}$. Sin embargo, aunque estas bacterias pueden causar daño directo a los tejidos periodontales, es la respuesta inmune del hospedero inducida ante los periodontopatógenos el principal determinante del carácter destructivo de la enfermedad ${ }^{(4,5)}$.

$P$. gingivalis es una bacteria patógena de alta prevalencia tanto en periodontitis crónica como agresiva ${ }^{(6,7,8)}$. La evidencia científica actual ha asociado a $P$. gingivalis con la destrucción activa del aparato de soporte periodontal(4) y con el inicio y severidad de ciertas enfermedades y condiciones sistémicas, tales como trastornos cardiovasculares y parto prematuro con bajo peso del neonato ${ }^{(1,9)}$.

En tanto, A. actinomycetemcomitans ha sido ampliamente asociada a periodontitis agresiva y se ha relacionado con el desarrollo de trastornos cardiovasculares, tal como la ateroesclerosis ${ }^{(10,11)}$.

Distintos serotipos se han descrito en bacterias que tienen la capacidad de inducir enfermedades infecciosas. En efecto, para Toxoplasma gondii y Escherichia coli se han descrito distintos serotipos, algunos de ellos asociados a enfermedad y otros a salud ${ }^{(12,13)}$. De igual forma, para $P$. gingivalis y $A$. actinomycetemcomitans se han propuesto distintos serotipos caracterizados por una virulencia diferente ${ }^{(14)}$.

El objetivo de esta revisión bibliográfica es analizar los principales factores de virulencia que expresan $P$. gingivalis y $A$. actinomycetemcomitans y como los distintos genotipos y/o serotipos descritos para ellos se podrían asociar con una inmunogenicidad y patogenicidad diferente durante las periodontitis. 
Patogenicidad y Virulencia Bacteriana

La virulencia se define como la capacidad de un microorganismo para producir una enfermedad o interferir con los procesos metabólicos o fisiológicos del hospedero(15,16). Un microorganismo virulento se caracteriza por expresar y producir metabolitos, toxinas, enzimas y componentes de la superficie o pared celular que le permiten evadir las barreras defensivas e invadir y sobrevivir en los tejidos y células del hospedero ${ }^{(17)}$. Poulin y Combes $^{(18)}$ definieron el concepto de virulencia en términos de factores de virulencia, refiriéndose a moléculas, componentes o estructuras microbianas que afectan al hospedero. De este modo, se ha propuesto que los factores de virulencia tienen múltiples funciones y se pueden agrupar en factores de adhesión, invasión, crecimiento y evasión de la respuesta inmune ${ }^{(16)}$.

Muchos factores de virulencia están codificados genéticamente y pueden expresarse en forma diferente entre distintas cepas de una misma especie bacteriana, así, se pueden definir distintos genotipos bacterianos sobre la base de un factor de virulencia particular.

Finalmente, distintos genotipos microbianos pueden generar un patrón de síntesis de anticuerpos distinto en el organismo infectado, definiéndose, sobre la base de esta respuesta sérica inducida, distintos serotipos bacterianos en una especie microbiana ${ }^{(19)}$.

Factores de Virulencia, Serotipos y Genotipos de Microorganismos Patógenos No Orales

E. coli es el microorganismo patógeno más frecuentemente detectado en infecciones del tracto genitourinario ${ }^{(12)}$. Para esta bacteria se han descrito distintos serotipos determinados por la variabilidad del antígeno $\mathrm{O}$ constituyente del lipopolisacárido (LPS) presente en la pared celular ${ }^{(20,21)}$. Una mayor virulencia se ha establecido para los serotipos

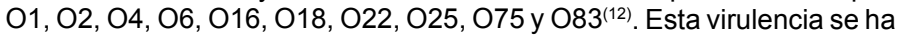
determinado en estudios clínicos en los que se correlacionó la presencia de infección con el serotipo bacteriano infectante, analizando además la expresión de otros factores de virulencia, tales como hemolisina (hly), factor de necrosis citotóxica 1 (cnf1), pili asociado a pielonefritis (pap) y adhesinas de la familia $S(s f a)^{(12)}$. En pacientes con infecciones genitourinarias, se han aislado cepas de $E$. coli para cuantificar la expresión de los genes antes descritos. Los serotipos 01, 06 y 015 fueron los más prevalentes $(12.2,10.2$ y $4.1 \%$ respectivamente) y la expresión de los genes pap, sfa, cnf y hly fue mayor en el serotipo O6 ${ }^{(12)}$.

Por otro lado, T. gondii, es un parásito intracelular que en humanos induce una amplia variedad de condiciones con distintas manifestaciones clínicas ${ }^{(13)}$. En pacientes inmunocompetentes las infecciones son prácticamente asintomáticas; mientras que, en sujetos inmunosuprimidos se desarrollan infecciones severas tal como la toxoplasmosis, enfermedad de carácter crónico que puede causar la muerte ${ }^{(13)}$. En T. gondii se describen 3 genotipos distintos basado en el polimorfismo de la porción hidrofílica $\mathrm{N}$-terminal del antígeno granular 5 (GRA5) y la región C-terminal hidrofilica del antígeno granular 6 (GRA6) y se denominan I, II y III(22,23). De ellos, se ha establecido que el serotipo II (GRA5-6) es el más frecuentemente detectado en toxoplasmosis humana ${ }^{(24)}$.

En conjunto, estos datos demuestran la variabilidad en la respuesta inmuno-inflamatoria inducida en humanos por $E$. coli y $T$. gondii, donde los serotipos y genotipos poseen distinta inmunogenicidad y patogenicidad. $P$. gingivalis y $A$. actinomycetemcomitans son consideradas las bacterias más frecuentemente detectadas en pacientes con periodontitis; sin embargo, son detectadas también en individuos sanos y en pacientes post-tratamiento periodontal. Así, es posible especular que los distintos genotipos y serotipos descritos para ellos son capaces de inducir una respuesta inmuno-inflamatoria diferente en el sujeto infectado y se podrían asociar a una patogenicidad variable que explicaría, al menos en parte, su detección tanto en salud como en enfermedad periodontal.

\section{Factores de Virulencia, Serotipos y Genotipos de Porphyromonas gingivalis}

$P$. gingivalis es un cocobacilo anaerobio facultativo Gram negativo, asociado a la etiología de las periodontitis. Este microorganismo expresa una serie de factores de virulencia, tales como fimbria, enzimas proteolíticas, hemaglutininas, LPS y cápsula ${ }^{(25)}$ y exhibe una diversidad genotípica y serotípica que permite una variabilidad intra-especie con la potencialidad de inducir inflamación y destrucción periodontal(26) (Tabla 1).
Tabla 1. Factores de virulencia de $P$. gingivalis. Variabilidad de genotipos y/o serotipos descritos para los distintos factores de virulencia descritos para $P$. gingivalis.

\begin{tabular}{|l|l|l|c|}
\hline \hline $\begin{array}{l}\text { Factor de } \\
\text { virulencia }\end{array}$ & Taxonomia & $\begin{array}{l}\text { Efectos } \\
\text { principales }\end{array}$ & Evidencia \\
\hline LPS & $01,02,03$ & $\begin{array}{l}\text { Reconocido por } \\
\text { receptores tipo Toll 2 y 4. } \\
\text { Induce secreción de } \\
\text { citoquinas proinflamatorias. }\end{array}$ & 24 y 42 \\
\hline Cápsula & $\begin{array}{l}\text { Ki, K2, K3, } \\
\text { K4,K5, K6 }\end{array}$ & $\begin{array}{l}\text { Induce diferenciación de } \\
\text { fenotipos } \\
\text { Th1, Th2, Th17 y Treg. }\end{array}$ & 5,14 y 73 \\
\hline Fimbria & $\mathrm{I}, \mathrm{Ib}, \mathrm{II}, \mathrm{III}$, & $\begin{array}{l}\text { Evasion de la fagocitosis. } \\
\text { Activación de RANKL. }\end{array}$ & 26 y 33 \\
\hline gen $\mathrm{kgp}$ & $\mathrm{I}, \mathrm{II}$ & Enzima proteolltica. & 25 \\
\hline gen rgpA & $\mathrm{A}, \mathrm{B}, \mathrm{C}$ & Enzima proteolltica. & 25 \\
\hline gingipaina & $\mathrm{R}, \mathrm{K}$ & $\begin{array}{l}\text { Edema. } \\
\text { Infiltración de neutrófilos. } \\
\text { Aumento del sangrado gingival. }\end{array}$ & 36 \\
\hline
\end{tabular}

Fimbria

La fimbria es una estructura filamentosa localizada en la superficie de $P$. gingivalis que le permite al microorganismo invadir los tejidos periodontales y colonizar la cavidad oral(27). Se compone de una subunidad proteica llamada fimbrilina, codificada por fimA, y otra subunidad llamada Mfa, codificada por $m f a 1^{(28)}$. Sobre la base del gen fimA, en $P$. gingivalis se han descrito 6 genotipos distintos, denominados I, Ib, II, III, IV y V(29).

En pacientes afectados con periodontitis crónica, los genotipos de $P$. gingivalis más frecuentemente detectados son el tipo II y IV ${ }^{(30)}$,

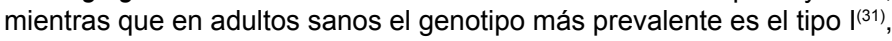
lo que implicaría una especificidad genotípica asociada al gen fimA entre salud y enfermedad periodontal.

En pacientes brasileños fumadores afectados de periodontitis crónica, la frecuencia de detección del genotipo fimA IV es $69.6 \%$, mientras que fimA II es detectado en el $28 \%$ de los $\operatorname{casos}^{(26)}$. En cambio, en la población de Noruega y Suiza, se ha establecido que $P$. gingivalis se aísla en el $71 \%$ de los pacientes con periodontitis crónica, detectándose el genotipo fimA II en $60 \%$ y fimA IV en $20 \%$ de ellos ${ }^{(32)}$.

La asociación entre presencia y severidad de las lesiones periodontales con el genotipo fimA detectado en ellas podría deberse a una variable patogenicidad bacteriana. En efecto, el genotipo fimA II es capaz de inhibir a los receptores que inducen la fagocitosis en macrófagos $^{(33)}$, además de inducir mayores niveles de expresión de RANKL, asociándose la mayor prevalencia de este genotipo con una mayor destrucción ósea alveolar y una mayor profundidad de las lesiones periodontales en pacientes afectados de periodontitis crónica ${ }^{(33)}$.

\section{Enzimas Proteolíticas}

Las gingipaínas son un grupo de proteasas producidas por $P$. gingivalis pertenecientes al grupo de trypsin-like cystein proteinases. Las gingipaínas $R g p A$ y $R g p B$ son codificadas por los genes $\operatorname{rgp} A$ y $\operatorname{rgp} B$, respectivamente, y son específicas para péptidos ricos en arginina (ArgXaa). Por otro lado, la gingipaína Kgp está codificada por el gen kgp y es específica para péptidos ricos en lisina (Lys-Xaa) ${ }^{(34)}$.

RgpA y Kgp son complejos que contienen dominios de separación catalítica y de adhesión hemaglutinínico; mientras que RgpB sólo presenta un dominio de catálisis ${ }^{(34,35)}$. RgpB determina el desarrollo del edema mediante la activación de la vía kalikreína/quinina y la infiltración por neutrófilos mediada por la activación de los factores quimiotácticos del complemento ${ }^{(36,37)}$. En cambio, Kgp y RgpA controlan el sangrado gingival a través de la degradación del fibrinógeno/fibrina(36).

RgpA y RgpB son capaces de inactivar citoquinas y sus receptores, estimular la agregación plaquetaria, atenuar la actividad antibacteriana de los neutrófilos por medio de la inhibición del receptor de LPS, incrementar la permeabilidad vascular y la apoptosis de los queratinocitos gingivales y destruir macrófagos CD14+(38). Kgp es capaz de promover la adhesión e invasión bacteriana in vitro ${ }^{(39)}$. En el fluido gingival crevicular de pacientes con periodontitis crónica RgpA y RgpB se asocian al aumento de los neutrófilos en los sitios periodontales infectados y Kgp, RgpA y RgpB al sangrado gingival ${ }^{(36,37)}$. 
Rgp se clasifica en dos isoenzimas, RgpA y RgpB, donde el gen $\operatorname{rgp} A$ no presenta variaciones genómicas mientras que $\operatorname{rgp} B$ posee 5 genotipos distintos. $K g p$, en tanto, presenta 2 genotipos diferentes denominados $k g p-1$ y kgp-2, determinados por el alineamiento de los nucleótidos en el dominio catalítico ${ }^{(38)}$. La literatura científica no ha revelado asociación entre estos genotipos y las condiciones periodontales ${ }^{(39,40)}$.

\section{Lipopolisacárido}

EI LPS de $P$. gingivalis contiene 3 componentes: polisacáridos (exterior), oligosacáridos (centro) y lípido A (interior)(41), siendo esta última la porción inmunogénica más activa ${ }^{(24)}$. Durante la enfermedad, $P$. gingivalis libera vesículas que contienen LPS, las que pueden invadir los tejidos periodontales y activar la producción de citoquinas en macrófagos, fibroblastos, queratinocitos y células endoteliales ${ }^{(42)}$, pudiendo eventualmente ser reconocidas por las células presentadoras de antígenos (APCs) con la capacidad de presentar sus antígenos a los linfocitos $T$ y desencadenar una respuesta inmune específica en el hospedero ${ }^{(24,42)}$. En efecto, fibroblastos gingivales humanos estimulados in vitro con LPS de $P$. gingivalis durante 48 horas expresan niveles incrementados, a nivel proteico y de mRNA, de IL-6 e IL-8 ${ }^{(24)}$.

EI LPS de $P$. gingivalis es reconocido por las APCs mediante receptores de tipo Toll (en inglés, toll-like receptor: TLR) 2 y $4^{(34,43)}$. Al analizar la respuesta inmune que el LPS de $P$. gingivalis es capaz de inducir en endoteliocitos y queratinocitos orales, se detectó un incremento en la secreción de IL-8 posterior a la neutralización de TLR-4; mientras que la neutralización de TLR-2 no produjo efectos en los niveles de IL$8^{(42)}$. Por otro lado, niveles incrementados de TNF- $\alpha$ fueron detectados cuando TLR-2 fue inhibida; mientras que la neutralización de TLR-4 no tuvo efectos ${ }^{(42)}$. Las células endoteliales activadas con LPS a través de TLR-4 y las células epiteliales activadas con LPS a través de TLR-2 presentan respuestas similares, secretando elevados niveles de IL-4, IL6, IL-6R, IL-10, IL-12p40 e IL-12p70(42). El reconocimiento diferencial de LPS mediado por TLR-2 y TLR-4 define una inmunogenicidad variable, la que podría explicarse por diferencias fenotípicas y genotípicas que definen su estructura, las que aún no han sido descritas.

\section{Polisacáridos Capsulares}

Existen macromoléculas en la superficie de las bacterias que confieren estabilidad estructural y que cumplen un rol importante en el reconocimiento e interacción con el hospedero. En bacterias patógenas, las macromoléculas de superficie también forman una barrera defensiva que le permiten evadir respuesta inmune ${ }^{(34)}$. En este contexto, los polisacáridos capsulares cumplen un rol importante en la mantención de la integridad de la célula en ambientes con alta presión inmune ${ }^{(34)}$.

La cápsula de $P$. gingivalis se compone principalmente de glucosa, glucosamina, galactosa, 2-acetamido-2-deoxy-D-glucosa, galactosamina y los ácidos galactosaminurónico, manurónico, glucorónico y galacturónico ${ }^{(44)}$, y sobre la base de su inmunogenicidad se han descrito 6 serotipos capsulares (K) diferentes, denominados $\mathrm{K} 1, \mathrm{~K} 2, \mathrm{~K} 3, \mathrm{~K} 4$, K5 y K6 $6^{(34,43,45)}$

En animales de experimentación, cuando los 6 serotipos capsulares se inocularon subcutáneamente, se observó un proceso infeccioso severo tipo flegmón, asociado a úlceras y/o necrosis de piel y muerte del animal. En contraste, al inocular una cepa de $P$. gingivalis carente de cápsula, sólo se observaron abscesos localizados, demostrándose la importancia virulenta de la cápsula extracelular ${ }^{(46)}$.

En modelos murinos, se evaluó la respuesta de los macrófagos ante los distintos serotipos $\mathrm{K}$ de $P$. gingivalis ${ }^{(44)}$, donde las quimioquinas MIP-2, JE/MCP-1, KC y RANTES se expresaron en niveles mayores en presencia del serotipo $\mathrm{K} 1$ en comparación a los otros serotipos, sugiriendo una respuesta quimiotáctica diferente inducida por los distintos serotipos $\mathrm{K}$ de $P$. gingivalis.

Por otro lado, cuando células dendríticas humanas diferenciadas in vitro a partir de monocitos periféricos fueron estimuladas con los distintos serotipos $\mathrm{K}$ de $\mathrm{P}$. gingivalis, se detectó que los serotipos $\mathrm{K} 1$ y K2 indujeron una mayor expresión de IL-1 $\beta$, IL-6, IL-12, IFN- $\gamma$, TNF- $\alpha$ y TNF- $\beta$ en comparación a los otros serotipos, demostrando una variable inmunogenicidad ${ }^{(14)}$

Factores de Virulencia, Serotipos y Genotipos de Aggregatibacter actinomycetemcomitans

A. actinomycetemcomitans es un cocobacilo, Gram negativo, capnofílico, no móvil, perteneciente a la familia de Pasteurellaceae, que ha sido implicado en la etiología de la periodontitis agresiva ${ }^{(47)}$
Tabla 2. Factores de virulencia de A. actinomycetemcomitans. Variabilidad de genotipos y/o serotipos descritos para los distintos factores de virulencia descritos para $A$. actinomycetemcomitans.

\begin{tabular}{|l|l|l|l|}
\hline $\begin{array}{l}\text { Factor de } \\
\text { virulencia }\end{array}$ & Taxonomia & $\begin{array}{l}\text { Efectos } \\
\text { principales }\end{array}$ & Evidencia \\
\hline Polisacárido O & a,b,c,d,e,f,¿g? & $\begin{array}{l}\text { Activación de células dendrí- } \\
\text { cas. Liberación de citoquinas } \\
\text { proinflamatorias. }\end{array}$ & 47,48 y 78 \\
\hline Leucotoxina & LtxA & $\begin{array}{l}\text { Induce la apoptosis de } \\
\text { neutrófilos }\end{array}$ & 52 \\
\hline EmaA & no aplica & Unión a colágeno I, III y IV. & 50 \\
\hline Adt & A,B,C & $\begin{array}{l}\text { Alteración de la morfología y } \\
\text { estructura celular. } \\
\text { Detención del cielo celular. }\end{array}$ & 62 y 63 \\
\hline
\end{tabular}

Diversos factores de virulencia se han descrito para $A$. actinomycetemcomitans, entre ellos, LPS, leucotoxina, fimbria, toxina distensora del citoesqueleto (Cdt), adhesinas epiteliales (Aae) y proteínas de adhesión e invasión celular (Omp100 y EmaA) ${ }^{(48-52)}$ (Tabla 2).

\section{Leucotoxina}

La capacidad que tiene $A$. actinomycetemcomitans para eliminar leucocitos está determinada por la presencia de leucotoxina $A$ $(\mathrm{LtXA})^{(53)}$, que se encuentra codificada por el gen Itx $A^{(54)}$. LtxA participa en la evasión de la respuesta inmune y la invasión tisular(54). Cuando A. actinomycetemcomitans invade los tejidos inflamados libera LtxA, adquiriendo la potencialidad de invadir neutrófilos e inducir su apoptosis en bajas concentraciones ${ }^{(52)}$ y su necrosis por disrupción de la membrana celular, en altas concentraciones ${ }^{(54)}$.

Cuatro genes para la leucotoxina han sido descritos, denominados ItxA, ItxB, ItxC y ItxD, donde ItxA es el gen estructural y ItxB, C y $D$ son genes requeridos para el transporte de la toxina ${ }^{(55,56)}$; sin embargo, no se han asociado a una variable inmunogenicidad o patogenicidad.

\section{Toxina distensora citoletal (Cdt)}

La toxina Cdt es una exotoxina proteica producida por bacterias Gram negativas que tiene la capacidad de detener el crecimiento de las células, alterar su morfología y eventualmente producir su muerte ${ }^{(57)}$ debido a su actividad DNAsa ${ }^{(58)}$. Análisis microbiológicos revelan que el $77 \%$ de los pacientes diagnosticados con periodontitis agresiva presentan $A$. actinomycetemcomitans $C d t+{ }^{(51)}$.

Cdt está conformada por la subunidad CdtB enzimáticamente activa y las subunidades CdtA y CdtC que permite la unión e invasión celular ${ }^{(59)}$ y produce daño en el DNA que resulta en la detención del ciclo celular entre las fases G2/M o G0/G1 en células del sistema inmune, tales como macrófagos y linfocitos $T^{(51)}$. Sin embargo, existe evidencia que demuestra que los fibroblastos del ligamento periodontal humano no son susceptibles a esta toxina ${ }^{(51,59,60)}$.

A. actinomycetemcomitans y Haemophilus ducreyi, agente etiológico del chancro, secretan Cdt, mostrando similitud estructural por sobre el $95 \%$ de la secuencia de aminoácidos ${ }^{(51,61)}$. En linfocitos $T$ activados con $\mathrm{Cdt}$ de $\mathrm{H}$. ducreyi o de $A$. actinomycetemcomitans se detectaron alteraciones estructurales celulares y un incremento en los niveles de RANKL a las 24 horas de estimulación, no observándose diferencias en los niveles de OPG(62).

En explantes gingivales de ratas y humanos expuestos a Cdt de $A$. actinomycetemcomitans se observó separación entre los distintos estratos epiteliales debido a alteraciones en las uniones intercelulares, sugiriendo daño estructural compatible con mayor invasión epitelial de $A$ actinomycetemcomitans $\mathrm{s}^{(63)}$.

Cdt tiene la capacidad de inhibir la producción de citoquinas proinflamatorias por APCs e induce la secreción de IL-1ß, IL-6, IL-8 e IFN-y por monocitos ${ }^{(64)}$. Al evaluar la reactividad de las distintas subunidades de Cdt en periodontitis crónica, agresiva localizada o agresiva generalizada, se observa que el $70.6 \%$ de los pacientes con periodontitis crónica tiene reactividad positiva a CdtB, el $100 \%$ de los pacientes con periodontitis agresiva generalizada fueron positivos para el CdtC y en periodontitis agresiva localizada la reactividad se distribuyó entre CdtA (33.3\%) y CdtB $(37.5 \%)^{(57)}$. En conjunto, estos resultados 
demuestran la existencia de una inmunogenicidad variable y asociación a la enfermedad entre las distintas subunidades estructurales de Cdt.

\section{Lipopolisacárido}

Las bacterias Gram negativas se caracterizan por tener en la membrana plasmática exterior una bicapa asimétrica de lipoproteínas: la capa interna compuesta principalmente de fosfolípidos y la externa de LPS, fosfolípidos y proteínas. EI LPS de A. actinomycetemcomitans se encuentra distribuido por toda la membrana exterior y es esencial para la mantención de la integridad bacteriana y el anclaje de otras proteínas ${ }^{(65,66)}$. Una molécula de LPS está conformada por el lípido A, que posee oligosacáridos que no se repiten dentro de su estructura central y de un polisacárido $\mathrm{O}$.

Seis serotipos han sido identificados: $a, b, c, d$, e y $f^{(67-70)}$, los que corresponden a distintos polisacáridos $\mathrm{O}$ estructural y antigénicamente diferentes ${ }^{(68)}$. Estos seis serotipos fueron descritos en tres linajes filogenéticos $^{(71)}$ donde dos corresponden a los serotipos b y c; mientras que el tercer linaje incluye a los serotipos $a, d$, e y $f^{(72)}$.

Inicialmente, se identificaron 6 antígenos y 24 patrones de aglutinación en 100 cepas de $A$. actinomycetemcomitans ${ }^{(67)}$. Al analizar la respuesta sérica de 16 cepas bacterianas en animales de experimentación, 12 antígenos en $A$. actinomycetemcomitans fueron detectados, revelando 3 patrones distintos de precipitación caracterizando los anticuerpos de la cepa 75 como serotipo a y los anticuerpos de las cepas Y4 y 67 como b y c, respectivamente ${ }^{(67)}$. Al evaluar la serología específica, se observó que las cepas NCTC 9710 y NCTC 9709 respondían de forma semejante a la cepa 67 , estableciéndose como serotipo c. Asimismo, la cepa ATCC 29523 se asoció al serotipo a y las cepas ATCC 29522 y ATCC 29524 al serotipo $b^{(67)}$.

De este modo, se demostró la existencia de 3 grupos de antígenos de $A$. actinomycetemcomitans.

Estos grupos serológicos se designaron como serotipos de acuerdo al International Code of Nomenclature of Bacteria and Viruses y la nomenclatura serológica del grupo Haemophilus. Así, se designaron a, b y c de acuerdo al orden de su identificación ${ }^{(67)}$. Estos resultados fueron concordantes con los obtenidos por King y Tatum ${ }^{(68)}$ quienes evaluaron el anti-suero de conejos inoculados con distintas cepas por medio de la precipitación por tubos capilares, describiendo 3 grupos séricos distintos. Posteriormente, 3 nuevos serotipos de $A$. actinomycetemcomitans han sido identificados, denominados $d$, e y $f^{(69,70)}$, y actualmente un posible serotipo $g$ se encuentra en estudio ${ }^{(73)}$

Al evaluar los anticuerpos presentes en el suero de pacientes afectados de periodontitis en presencia de $A$. actinomycetemcomitans se estableció que el serotipo $b$ induce una mayor síntesis y secreción de $\lg G$, IgM e $\lg A$, mientras que los serotipos a y $c$ no evidenciaron diferencias ${ }^{(68)}$.

Los serotipos de $A$. actinomycetemcomitans más frecuentemente detectados en la cavidad oral en humanos son $a, b$ y $c$, siendo el serotipo $b$ el detectado con mayor frecuencia en pacientes afectados de periodontitis agresiva ${ }^{(74)}$. La predominancia del serotipo b en pacientes con periodontitis agresiva sugiere un alto potencial patogénico, aún no establecido ${ }^{(48)}$. Se ha reportado que el serotipo $b$ de $A$. actinomycetemcomitans resiste a la fagocitosis ${ }^{(74)}$ y evade la respuesta inmune de los macrófagos y neutrófilos ${ }^{(47)}$, mientras que los serotipos a y c son capaces de inducir la síntesis de IL-1 $\beta$ en macrófagos ${ }^{(75)}$.

En concordancia con lo anterior, al evaluar el efecto de los 3 serotipos más prevalentes ( $a, b$ y $c$ ) en células epiteliales de biopsias gingivales de pacientes afectados de periodontitis, se ha observado que el serotipo b es el más prevalente en periodontitis agresiva ${ }^{(76)}$ y es capaz de producir microvesículas citotóxicas ${ }^{(77)}$, a diferencia de los otros serotipos $^{(48)}$

\section{CONCLUSIÓN}

P. gingivalis y $A$. actinomycetemcomitans son las bacterias periodontopatógenas más frecuentemente detectadas en pacientes con periodontitis. Ambas bacterias poseen distintos factores de virulencia por medio de los cuales pueden adherirse e invadir las células y tejidos del hospedero e inducir una respuesta inmuno-inflamatoria que puede causar la destrucción de la inserción periodontal ${ }^{(1)}$.

Los factores de virulencia los podemos agrupar según las propiedades que le otorga al microorganismo como: factores de adhesión, multiplicación, invasión y evasión. Tanto el pili como LPS son factores que permiten a las bacterias adherirse a moléculas presentes en la superficie de las células, otras bacterias o macromoléculas. Una vez invadidas la células y tejidos del hospedero, la bacteria puede multiplicarse ${ }^{(50)}$. La fimbria se puede considerar un factor de invasión tisular, mientras que la LtxA, los polisacáridos de superficie y la cápsula son factores de evasión de la respuesta inmune ${ }^{(50)}$. Las modificaciones post-traduccionales de los componentes de la superficie bacteriana, como las gingipainas, fimbria y LPS, desempeñan un rol funcional en la regulación de la virulencia del microorganismo.

En $P$. gingivalis, los factores de virulencia más importantes son la fimbria, el LPS y la cápsula, mientras que en A. actinomycetemcomitans son la LxtA, la toxina Cdt y el LPS ${ }^{(5,14,32,42,52,63)}$.

EI LPS y la cápsula bacteriana son importantes en $P$. gingivalis y $A$. actinomycetemcomitans dado que inducen variabilidad en la respuesta inmune desplegada por el hospedero. En efecto, sobre la base de la imunogenicidad de los polisacáridos que conforman la cápsula de $P$. gingivalis, como del polisacárido $O$ de $A$. actinomycetemcomitans, se describen distintos serotipos bacterianos, los que podrían asociarse a un distinto potencial virulento, a una variable patogenicidad y a una asociación diferente en los estados de salud y enfermedad periodontal ${ }^{(5,14,77)}$.

\section{CONFLICTOS DE INTERÉS}

Los autores declaran no tener conflictos de interés.

\section{REFERENCIAS BIBLIOGRÁFICAS}

1. Offenbacher $S$, Lieff $S$, Boggess KA, Murtha AP, Madianos PN, Champagne CM, McKaig RG, Jared HL, Mauriello SM, Auten RL, Herbert WN, Beck JD. Maternal periodontitis and prematurity. Part I: Obstetric outcome of prematurity and growth restriction. Ann Periodontol, 2001; 6: 164-174.

2. Tonetti MS, Imboden MA, Lan NP. Neutrophil migration into the gingival sulcus is associated with transepithelial gradients of interleukin- 8 and ICAM-1. J Periodontol, 1998; 69: 1139-1147.

3. Socransky SS, Haffajee AD. Dental biofilms: Difficult therapeutic targets. Periodontology 2000, 2002; 28: 12-55.

4. Slots J, Bragd L, Wikstrom M, Dahle G. The occurrence of Actinobacillus actinomycetemcomitans, Bacteroides gingivalis and Bacteroides intermedius in destructive periodontal disease in adults. $J$ Clin Periodontol, 1986; 13: 570-577.

5. van Winkelhoff AJ, Loos BG, van der Reijden WA, van der Velden U. Spain Porphyromonas gingivalis, Bacteroides forsythus and other putative periodontal pathogens in subjects with and without periodontal destruction. J Clin Periodontol, 2002; 29: 1023-1028.
6. Page RC, Offenbacher S, Schroeder HE, Seymour GJ, Kornman KS. Advances in the pathogenesis of periodontitis: Summary of developments, clinical implications and future directions. Periodontol 2000, 1997; 14: 216248.

7. Dutzan N, Gamonal J, SilvaA, Sanz M, Vernal R. Over-expression of forkhead box $\mathrm{P} 3$ and its association with receptor activator of nuclear factor- $\kappa \beta$ ligand, interleukin (IL)-17, IL-10 and transforming growth factor $\beta$ during the progression of chronic periodontitis. J Clin Periodontol, 2009; 36: 396 -403.

8. Silva N, Dutzan N, Hernández M, Dezerega A, Rivera O, Aguillón $\mathrm{JC}$ et al. Characterization of progressive periodontal lesions in chronic periodontitis patients: Levels of chemokines, cytokines, matrix metalloproteinase-13, periodontal pathogens and inflammatory cells. J Clin Periodontol, 2008; 35: 206-214.

9. Meurman JH, Sanz M, Janket SJ. Oral health, atherosclerosis, and cardiovascular disease. Crit Rev Oral Biol Med, 2004; 15: 403-413.

10. Asikainen S, Lai CH, Alaluusua S, Slots J. Distribution of Actinobacillus actinomycetemcomitans serotypes in periodontal health and disease. Oral Microbiol Immunol, 1991; 6: 115-118. 
11. Zhang T, Kurita-Ochiai T, Hashizume T, Du Y, Ogushi S, Yamamoto M. Aggregatibacter actinomycetemcomitans accelerates atherosclerosis with an increase in atrherogenic factors in spontaneously hyperlipidemic mice. Immunol Med Microbiol, 2010; 59: 143-151.

12. Emamghorashi F, Farchad S, Kalani M. Relashionship between $O$ serotype and virulent genes in Escherichia coli causing urinary tratc infections. Ir J Kidney Diseases, 2011; 5(4): 234-237.

13. Morisset S, Peyron F, Lobry JR, Garweg J, Ferrandiz J, Musset K, GomezMarin JE, de la Torre A, Demar M, Carme B, Mercier C, Garin JF, CesbronDelaw MF. Serotyping of Toxoplasma gondii: Striking homogeneous patter between symptomatic and asymptomatic infections within Europe and South America. Microbes and Infection, 2008; 10: 742 -747.

14. Vernal R, León R, Silva A, van Winkelhoff AJ, García-Sanz JA, Sanz M. Differential cytokine expression by human dendritic cells in response to different Porphyromonas gingivalis capsular serotypes. J Clin Periodontol, 2009; 36: 823-829.

15. Slots J. Actinobacillus actinomycetemcomitans and Porphyromonas gingivalis in periodontal disease: Introduction. Periodontology 2000, 1999; 20: 7-13.

16. Holt SC, Ebersole JL. The oral spirochetes: Their ecology and pathogenesis. In: Radolf J, Lukehart S, editors. Molecular Biology and Pathogenesis of Treponemal Infections. Norfolk, UK: Horizon Scientific Press, 2005

17. Holt SC, Kesavalu L, Walker S, Genco CA. Virulence factors in Porphyromonas gingivalis. Periodontol 2000, 1999; 20: 168-238.

18. Poulin R, Combes C. The concept of virulence: Interpretations and implications. Parasitol Today, 1999; 15: 474-475.

19. Ebersole JL, Sandoval MN, Steffen MJ, Cappelli D. Serum-Antibody in Actinobacillus actinomycetemcomitans-Infected pactients with periodontal disease. Infection and Immunity, 1991; 59(5): 1795-1802. 20. Kauffmann F. Ueber neue themolabile Korperantigene der colibakterien. Acta Pathol Microbiol Scand, 1943; 20: 21-44.

21. Gruneberg RN. Antibiotic sensitivities of urinary pathogens, 1971-82. J Antimicrob Chemother, 1984; 14: 17-23.

22. Howe DK, Sibley LD. Toxoplasma gondii comprises three clonal lineages: Correlation of parasite genotype with human disease. J Infect Dis, 1995; 172: 1561-1566.

23. Sibley LD, Mordue DG, Su C, Robben PM, Howe DK. Genetic approaches to studying virulence and pathogenesis in Toxoplasma gondii. R Soc Lond B Biol Sci, 2002; 357: 81-88.

24. Herath TD, Wang Y, Seneviratne CJ, Lu Q, Darveau RP, Wang CY, Jin L. Porphyromonas gingivalis lipopolysaccharide lipid A heterogeneity differentially modulates the expression of IL- 6 and IL-8 in human gingival fibroblasts. J Clin Periodontol, 2011; 38: 694-701.

25. Yoshino T, Laine M, van Winkelhoff AJ, Dahlén G. Genotype variation and capsular serotypes of Porphyromonas gingivalis from chronic periodontitis and periodontal abcsesses. FEMS Microbiol Lett, 2007; 270: 75-81.

26. Teixeira SRL, Mattarazo F, Ferez M, Figueredo LC, Faveri M, Simionato MRL, Mayer MPA. Quantification of Prohyromonas gingivalis and fimA genotypes in smoker chronic periodontitis. J Clin Periodontol, 2009; 26: 482 $-487$.

27. Hiramine H, Watanabe K, Hamada N, Umemoto T. Porphyromonas gingivalis $67-\mathrm{kDa}$ fimbriae induced cytokine production and osteoclast differentiation utilizing TLR2. FEMS Microbiology Letters, 2003; 229: 4955.

28. Kuboniwa M, Inaba H, Amano A. Genotyping to distinguish microbial pathogenicity in periodontitis. Periodontology 2000, 2010; 54: 136-159.

29. Amano A, Nakagawa I, Okahashi N, Hamada N. Variations of Porphyromonas gingivalis fimbriae in relation to microbial pathogenesis. J Periodontal Res, 2004; 39: 136-142.

30. Enersen M, Olsen I, Kvalheim O, Caugant DA. fimA genotypes and multilocus sequence types of Porphyromonas gingivalis from patients with periodontitis. J Clin Microbiol, 2008; 46: 31-42.

31. Amano A, Kuboniwa M, Nakagawa I, Akiyama S, Morisaki I, Hamada S. Prevalence of specific genotypes of Porphyromonas gingivalis fimA and periodontal health status. J Dent Res, 2000; 79: 1664-1668.

32. van der Ploeg JR, Giertsen E, Lü din B, Mörgeli C, Zinkernagel AS, Gmur R. Quantitative detection of Porphyromonas gingivalis fimA genotypes in dental plaque. FEMS Microbiology Letters, 2004; 232: 3137 .

33. Wang M, Liang Sm Hosur K, Domon H, Yoshimura F, Amano A, Hajishengallis $G$. Differential virulence and innate interactions of Type I and II fimbrial genotypes of Porphyromonas gingivalis. Oral Microbiol Immunol, 2009; 24(6): 478-484.
34. Poltorak A, He X, Smirnova I et al. Defective LPS signaling in $\mathrm{C} 3 \mathrm{H} /$ $\mathrm{HeJ}$ and C57BL/10ScCr mice: Mutations in TLR4 gene. Science, 1998 282: 2085-2088.

35. Osbourne DO, Aruni W, Roy F, Perry C, Sandberg L, Muthiah A Fletcher $\mathrm{H}$. Role of vimA cell surface biogenesis in Porphyromonas gingivalis. Microbiology, 2010; 156: 2180-2193.

36. Travis J, Pike R, Imamura T, Potempa J. Porphyromonas gingivalis proteinases as virulence factors in the development of periodontitis. $J$ Periodontal Res, 1997; 32(1): 120-125.

37. Imamura T. The role of gingipains in the pathogenesis of periodontal disease. J Periodontol, 2003; 71(1): 111-118.

38. Stathopoulou PG, Galicia JC, Benakanakere MR, García CA, Potempa J, Kinane DF. Porphyromonas gingivalis induce apotosis in human gingival epitelial cells through a gingipain-dependent mechanism. BMC Microbiol, 2009; 9: 107.

39. Beikler T, Peters U, Prior K, Ejhmke B, Flemmig TF. Sequence variations in rgpA and rgpB of Porphyromonas gingivalis in periodontitis. J Periodontal Res, 2005; 40(3): 193-198.

40. Kubinowa M, Inaba H, Amano A. Genotyping to distinguish microbial pathogenicity in periodontitis. Periodontol 2000, 2000; 54(1): 136-159.

41. Dixon DR, Darveau RP. Lipopolysaccharide heterogeneity: Innate host responses to bacterial modification of lipid a structure. J Dent Res, 2005; 84: 584-595.

42. Kocgozlu L, Elkaim R, Tenenbaum $\mathrm{H}$, Werner $\mathrm{S}$. Variable cell responses to $P$. gingivalis lipopolysaccharide. J Dent Res, 2009; 88(8): 741-745.

43. Kikkert R, Laine ML, Aarden LA, van Winkelhoff AJ. Activation of toll-like receptors 2 and 4 by gram-negative periodontal bacteria. Oral Microbiol Immunol, 2007; 22: 145-151.

44. d'Empaire G, Baer MT, Gibson FC. K1 serotype capsular polysaccharide of Porphyromonas gingivalis elicits chemokine production from murine macrophages that facilitates cell migration. Infect Immun, 2006; 74(11): 6236-6243

45. Laine ML, Appelmelk BJ, van Winkelhoff. Novel polysaccharide capsular serotypes in Porphyromonas gingivalis. J Periodontal Res, 1996; 31(4): 278-284.

46. Laine ML, van Winkelhoff AJ. Virulence of six capsular serotypes of Porphyromonas gingivalis in a mouse model. Oral Microbiol Immunol, 1998; 13(5): 322-325

47. Suzuki N, Nakano Y, Yoshida Y, Ikeda D, Koga T. Identification of Actinobacillus actinomycetemcomitans serotypes by multiplex PCR. J Clin Microbiol, 2001; 39: 2002-2005.

48. Shimada T, Sugano N, Nishihara R, Suzuki K, Tanaka H, Ito K. Differential effects of five Aggregatibacter actinomycetemcomitans strains on gingival epithelial cells. Oral Microbiol Immunol, 2008; 23: 455-458. 49. Bosshardt DD, Lang NP. The junctional epithelium: From health to disease. J Dent Res, 2005; 85: 9-20.

50. Iniesta M, Herrera D, Serrano J, Sanz M. Análisis de los factores de virulencia de los patógenos de asociación fuerte con la periodontitis: Aggregatibacter actinomycetemcomitans, Porphyromonas gingivalis y Tannerella forsythia. Periodoncia y Oseointegración, 2008; 18(2): 109115.

51. Xynogala I, Volgina A, DiRienzo JM, Korostoff J. Evaluation of the humoral immune response to the cytolethal distending toxin of Aggregatibacter actinomycetemcomitans in subjects with localized aggresive periodontitis. Oral Microbiol Immunol, 2009; 24(2): 116-123.

52. Kachlany SC. Aggregatibacter actinomycetemcomitans leukotoxin: From threat to therapy. J Dent Research, 2010; 89(6): 561-570.

53. Baehni PC, Tsai CC, McArthur WP, Hammond BF, Shenker BJ, Taichman NS. Leukotoxic activity in different strains of the bacterium Actinobacillus actinomycetemcomitans isolated from juvenile periodontitis in man. Arch Oral Biol, 1981; 26: 671-676.

54. Diaz R, Ghofaily LA, Patel J, Balashova NV, Freitas AC, Labib I et al. Characterization of leukotoxin from a clinical strain of Actinobacillus actinomycetemcomitans. Microb Pathog, 2006; 40: 48-55.

55. Kraig E, Dailey T, Kolodrubetz D. Nucleotide sequence of the leucotoxin gene from Actinobacillus actinomycetemcomitans: Homology to the alphahemolysin/leukotoxin gene family. Infect Immun, 1990; 58(4): 920-929. 56. Lally ET, Golub EE, Kieba IR, Taichman NS, Rosenbloom J, Rosenbloom JC, Gibson CW, Demuth DR. Analysis of the Actinobacillus actinomycetemcomitans leukotoxin gene. Delineation of unique features and comparison to homologous toxins. J Biol Chem, 1989; 264(26): 1545115456.

57. Heywood W, Henderson B, Nair SP. Cytolethal distending toxin Creating a gap in the cell cycle. J Med Microbiol, 2005; 54: 207-216.

58. Frisan T, Cortes-Bratti X, Chaves-Olarte E, Stenerlow B, Thelestam 
M. The hemophilus ducreyi cytolethal distending toxin induces DNA double - strand breaks and promotes ATM-dependent activation of RhoA. Cell Microbiol, 2003; 5: 965-707.

59. Ando ES, De-Gennaro LA, Faveri M, Feres M, DiRienzo JM, Mater MPA. Immune response to cytolethal distending toxin of Aggregatibacter actinomycetemcomitans in peridontitis patiens. J Periodontal Res, 2010; 45: 471-480.

60. Smith JL, Bayles DO. The contribution of cytolethal distending toxin to bacterial pathogenesis. Crit Rev Microbiol, 2006; 32: 227-248.

61. Fabris AS, DiRienzo JM, Wikstrom M, Mayer MP. Detection of cytolethal distending toxin activity and cdt genes in Actinobacillus actinomycetemcomitans isolates from geographically diverse populations. Oral Microbiol Immunol, 2002; 17: 231-238.

62. Belibasakis GN, Brage M, Lagergard T, Johansson A. Cytolethal distending toxin upregulates RANKL expression in Jurkat T-cells. Journal Compilation, 2008; 116: 499-506.

63. Damek-Poprawa M, Haris M, Volgina A, Korostoff J, DiRienzo JM. Cytolethal distending toxin damages the oral epithelium of gingival explants. J Dent Res, 2011; 90(7): 874-879.

64. Akifusa S, Poole S, Lewthwaite J, Henderson B, Nair SP. Recombinant Actinobacillus actinomycetemcomitans cytolethal distending toxin proteins are required to interact to inhibit human cell cycle progression and to stimulate human leukocyte cytokine synthesis. Infect Immun, 2001; 69: 5925-5930.

65. Holt SC, Tanner AC, Socransky SS. Morphology and ultrastructure of oral strains of Actinobacillus actinomycetemcomitans and Haemophilus aphrophilus. Infect Immun, 1980; 30: 588-600.

66. Zambon JJ, DeLuca C, Slots J, Genco RJ. Studies of leukotoxin from Actinobacillus actinomycetemcomitans using the promyelocytic HL-60 cell line. Infect Immun, 1983; 40: 205-212.

67. Zambon JJ, Slots J, Genco R. Serology of oral Actinobacillus actinomycetemcomitans and serotipe distribution in human periodontal disease. Infect Immun, 1983; 41(1): 19-27.

68. King EO, Tatum HW. Actinobacillus actinomycetemcomitans and Haemophilus aphrophilus. J Infect Dis, 1962; 111: 85-94.

69. Kaplan JB, Perry MB, MacLean LL, Furgang D, Wilson ME, Fine DH. Structural and genetic analyses of $O$ polysaccharide from Actinobacillus actinomycetemcomitans serotype f. Infect Immun, 2001; 69(9): 5375-5384.
70. Gmür $\mathrm{R}$, McNabb $\mathrm{H}$, van Steenbergen TJM, Baehni $\mathrm{P}$, Mombelli A, van Winkelhoff AJ, Guggenheim B. Seroclassification of hitherto nontypeable Actinobacillus actinomycetemcomitans strains: Evidence for a new serotype e. Oral Microbiol Immunol, 1993; 8: 116-120.

71. Kaplan JB, Schreiner HC, Furgang D, Fine DH. Population structure and genetic diversity of Actinobacillus actinomycetemcomitans strains isolated from localized juvenile periodontitis patients. J Clin Microbiol, 2002; 40: 1181-1187.

72. Poulsen K, Theilade E, Lally ET, Demuth DR, Kilian M. Population structure of Actinobacillus actinomycetemcomitans: A framework for studies of disease-associated properties. Microbiology, 1994; 140: 20492060.

73. Takada K, Saito M, Tsuzukibashi O, Kawashima Y, Ishida S, Hirasawa M. Characterization of a new serotype $\mathrm{g}$ isolate of Aggregatibacter actinomycetemcomitans. Mol Oral Microbiol, 2010; 25(3): 200-206.

74. Asikainen S, Lai CH, Alaluusua S, Slots J. Distribution of Actinobacillus actinomycetemcomitans serotypes in periodontal health and disease. Oral Microbiol Immunol, 1991; 6: 115-118.

75. Yamaguchi N, Kawasaki M, Yamashita Y, Nakashima K, Koga T. Role of the capsular polysaccharide-like serotypespecific antigen in resistance of Actinobacillus actinomycetemcomitans to phagocytosis by human polymorphonuclear leukocytes. Infect Immun, 1995; 63: 4589-4594.

76. Cortelli JR, Aquino DR, Cortelli SC, Roman-Torres CVG, Franco GCN, Gomez RS, Batista LH, Costa FO. Aggregatibacter actinomycetemcomitans serotypes infections and periodontal conditions: A two way assesment. Eur J Clin Microbiol Infect Dis, 2011; en impresión. 77. Lai $\mathrm{CH}$, Listgarten MA, Hammond BF. Comparative ultrastructure of leucotoxic and non-leucotoxic strains of Actinobacillus actinomycetemcomitans. J Periodontal Res, 1981; 16: 379-389.

78. Vernal T, León R, Herrera D, García-Sanz JA, Silva A, Sanz M Variability in the response of human dendritic cells stimulated with Porphyromonas gingivalis or Aggregatibacter actinomycetemcomitans. $J$ Periodontol Res, 2008; 43: 689-697. 